\title{
Low Loss Transmission Line for a 3.4-kW, 93-GHz Gyro-Traveling-Wave Amplifier
}

\author{
Craig R. Donaldson, Liang Zhang, Wenlong He, Adrian W. Cross, Kevin Ronald, and Colin G. Whyte
}

\begin{abstract}
In this paper a transmission line system for the propagation of millimeter wave radiation is presented. The full system includes a $\mathrm{TE}_{11}-\mathrm{TE}_{01}$ mode converter, waveguide tapers, mitre bends and many straight sections. The design of each of these components is described and optimized simulation results are given. The mode converter shows a greater than $96 \%$ mode conversion efficiency can be achieved over a $2 \%$ bandwidth, at W-band. The mitre bends demonstrate a transmission loss of $0.04 \mathrm{~dB}$ each over the same bandwidth, when they are configured to introduce a mixture of higher order waveguide modes before the reflecting surface. An example transmission line system with a propagation length of $20 \mathrm{~m}$, inclusive of four 90 degree bends with an OFHC copper waveguide material, was studied over a 90-96 GHz frequency range and showed a $0.84 \mathrm{~dB}$ transmission loss at $93 \mathrm{GHz}$.
\end{abstract}

Index Terms-Transmission line, mode converter, mitre bend, gyro-traveling wave tube.

\section{INTRODUCTION}

$\mathbf{M}$ ILLIMETER-wave (MMW) radiation in W-band, at the $\mathrm{kW}$ power level, is desirable for a number of applications. With Electron Paramagnetic Resonance (EPR) the increased peak millimeter-wave power improves spatial resolution, and shortens the imaging times [1]. Nuclear fusion research experiments require quasi $\mathrm{CW}$ millimeter-wave sources at multi-megawatt level power to heat a fusion plasma [2]. The sources for these applications must often be sited a significant distance from the point of use. The physical size of the source, electronic noise from high-voltage power supplies and stray magnetic fields are all reasons to locate the source some distance from the point of application. Transmitting the radiation to the point of use with low insertion loss while maintaining the correct mode pattern becomes an important issue.

Propagating the MMW in free space is the simplest solution, however the transmitting apertures must be large in order to control losses due to diffraction therefore maintaining a propagating mode in a metallic waveguide structure is a more practical method. The $\mathrm{TE}_{01}$ mode in a circular waveguide and the $\mathrm{HE}_{11}$ mode in a corrugated waveguide [3]-[5] are

This work was supported by STFC UK (research Grant No. ST/T003227/1) and EPSRC (research Grant No. EP/K029746/1).

C. R. Donaldson, L. Zhang, A. W. Cross, K. Ronald and C. G. Whute are with the Department of Physics, SUPA, University of Strathclyde, Glasgow, G4 0NG, UK (e-mail: craig.donaldson@strath.ac.uk).

W. He is with the College of Electronic Science and Technology, Shenzhen University, Shenzhen, China. suitable options with attractive properties, such as a centrally located wave power within the waveguide which reduces the field strength at the waveguide wall and minimizes conduction losses. It is also possible to couple to either mode with low insertion loss. The $\mathrm{HE}_{11}$ mode allows propagation in linear or circular polarization, has a quasi-optical far-field radiation pattern useful for many applications, and shows broad operational bandwidth (example, 100-160 GHz for application in the International Thermonuclear Experimental Reactor, ITER [6]). The $\mathrm{HE}_{11}$ transmission line has been widely used in plasma fusion and DNP systems [7], however, the corrugated waveguide is relatively complicated and expensive to manufacture, especially at higher operating frequencies where dimensions become smaller and tolerances tighter. The $\mathrm{TE}_{01}$ mode also has a symmetric, and centrally located, electric field with low loss, yet it propagates in a smooth circular waveguide. An argument can be made that lower manufacturing costs and more robust accommodation of manufacturing tolerances supports the use of $\mathrm{TE}_{01}$ transmission systems.

The proposed transmission line system includes: (1) a mode converter to convert output mode of the source in to propagating mode of the transmission line system; (2) waveguide tapers to match waveguide diameters; (3) waveguide bends to allow for directional changes; (4) straight waveguide sections with low loss.

A mitre bend can introduce mode conversion and thereby loss of power in the desired modes. In 1993 it was proposed that these negative effects could be significantly reduced through conversion of incoming mode $\left(\mathrm{TE}_{01}\right)$ to higher order $\mathrm{TE}_{0 n}$ modes [8]. The improved design, incorporating a mode converter, was measured at W-band frequencies $(93-98 \mathrm{GHz})$ [9]. Further work at Ka-band focused on a transmission line system with a mode converter $\left(\mathrm{TE}_{11}-\mathrm{TE}_{01}\right)$ and a series of the improved mitre bends [10]. A significant reduction in the transmission loss of 15 times was achieved as compared to the conventional mitre bend. This approach has also been utilized in applications such as the resonant ring for the testing of components with high power microwaves [11]. A design of mitre bend with a $\mathrm{TE}_{03}$ mode dominant, with a weaker mixture of $\mathrm{TE}_{04}$ and $\mathrm{TE}_{02}$ modes, has been used for overmoded Gyrotrons [8].

This paper presents a $20 \mathrm{~m}\left(6250 \lambda_{0}\right)$ long transmission line system for an application where the source is sited away from the load or antenna or point of use. The paper is organized as follows. The design of the mode converter, waveguide taper and mitre bend, are presented in detail. A transmission line 


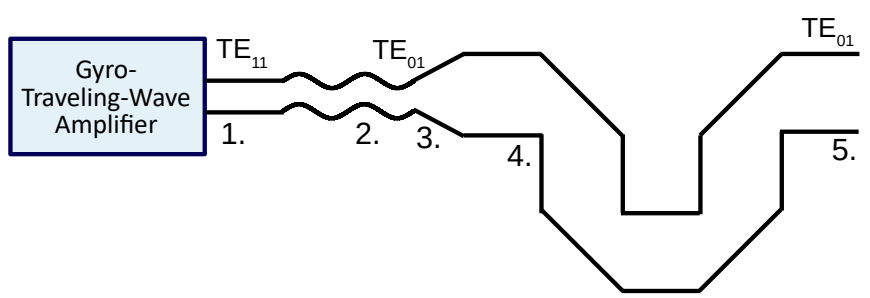

Fig. 1: Transmission system showing 1) circular waveguide with $\mathrm{TE}_{11}$ mode, 2) $\mathrm{TE}_{11}$ to $\mathrm{TE}_{01}$ mode converter, 3) linear taper, 4) mitre bends, and 5) output to application.

system suitable for a W-band gyrotron traveling wave amplifier (gyro-TWA) [12], [13], with circularly polarized $\mathrm{TE}_{11}$ output is presented.

\section{COMPONENTS IN A TRANSMISSION LINE SYSTEM}

The general scheme of the transmission line system is shown in Fig. 1. The output from the MMW radiation source is transmitted some distance to the end application through this system. Four mitre bends are included in the proposed system to show the flexibility of the system, for example where obstructions prevent a direct transmission path, as often occurs in complex systems.

\section{A. Mode Converter}

Mode converters of many forms can be designed depending on the characteristics of the desired input and output modes [14]-[16]. Even for the same conversion, (for example the $\mathrm{TE}_{11}$ to $\mathrm{TE}_{01}$ mode in circular waveguide), there are different ways to realize the mode converter [17], [18].

In this paper, a mode conversion approach based on mode coupling theory is used. The surface profile of such a mode converter has the following expression in cylindrical coordinates [19].

$$
R(z, \theta)=R_{0}+\varepsilon R_{0} \cos \left(2 \pi z / \lambda_{B}\right) \cos \left(m_{B} \theta\right)
$$

where $R_{0}$ is the average radius, $\varepsilon$ is the maximum corrugation amplitude deviation from the average radius, $\lambda_{B}$ is the beat wavelength, and $m_{B}$ is the periodicity in the azimuthal direction. The mode conversion can be examined from the dispersion curves of the incident mode (mode 1) and the spatial harmonic of the output mode (mode 2) (Fig. 2). At the intersection point, where the Bragg resonance conditions are satisfied, the two modes have strong coupling and can convert the energy of the input mode to the output mode [20]. Theoretically, this kind of mode converter is able to achieve mode coupling between any two modes.

To convert $\mathrm{TE}_{11}$ mode to $\mathrm{TE}_{01}$ mode at a center frequency of $93 \mathrm{GHz}$, the initial geometry parameters of the beat-wave mode converter can be derived from the mode coupling equation based on perturbation theory [21]-[23]. This yields a first pass design which can then be optimized to meet the requirements for coupling coefficient or bandwidth. For example, reducing $\varepsilon$ results in a longer structure with

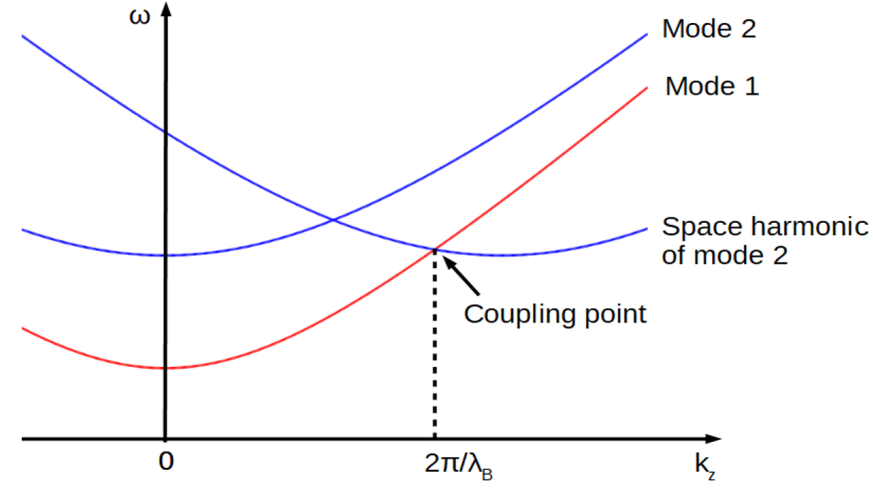

Fig. 2: Dispersion curves of the coupling modes.

larger bandwidth. Fig. 3 shows the simulation results with the geometry parameters, $R_{0}=2.50 \mathrm{~mm}, \varepsilon=0.15, \lambda_{B}=10.65 \mathrm{~mm}$, and the overall length $34.30 \mathrm{~mm}$. The central frequency mode conversion is $99.1 \%$ while at the top and tail of the $2 \%$ pass band the conversion is $98.0 \%$ and $96.9 \%$ respectively.

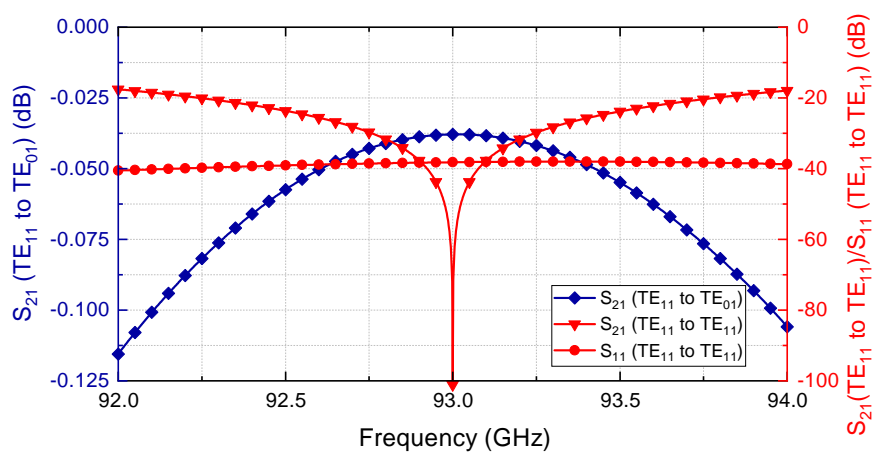

(a)

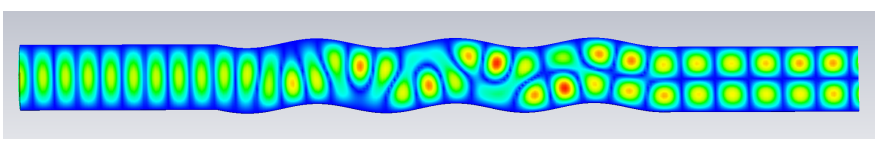

(b)

Fig. 3: Scattering parameters of the mode converter (a), and the field pattern inside the mode converter at the center frequency (b).

The length of the mode converter and its effect on the mode conversion efficiency was investigated, data shown in Fig. 4. It is seen that with the higher number of beat wavelengths the bandwidth reduces, however the central mode conversion increases. Therefore, the choice of length will be a trade-off between requirements of the high/low ends of the bandwidth and the mode purity required at the central frequency.

\section{B. Mitre Bend for TE 01 Mode and Waveguide Taper}

The geometry of a 90 degree mitre bend is shown in Fig. 5(a). It consists of two straight waveguides, at a 90 degree angle to each other with a 45 degree sloped surface.

The transmission of the operating $\mathrm{TE}_{01}$ mode is entirely dependent on the diameter of the waveguide (d) and the distance between its bending point and the sloped surface (b). 


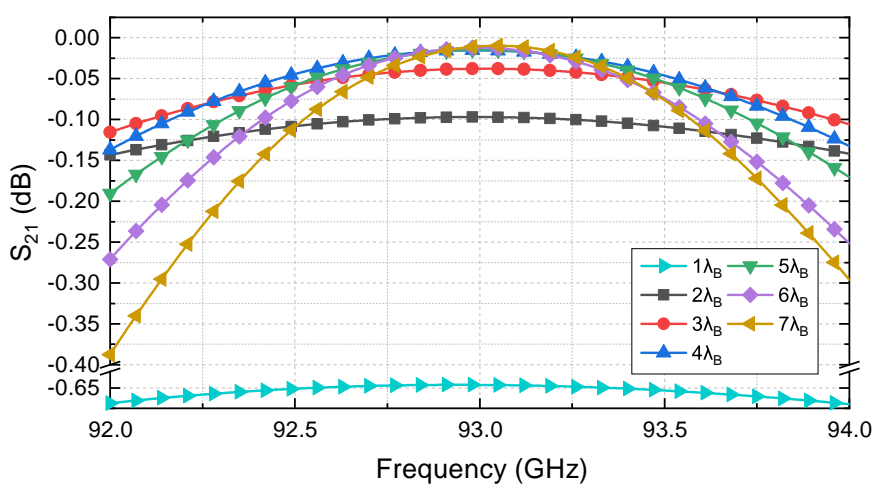

Fig. 4: Mode conversion at different number of beat wavelengths.

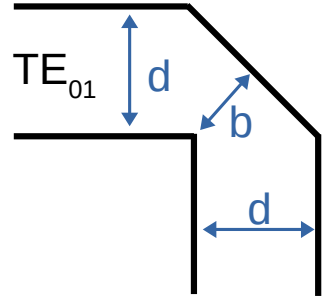

(a)

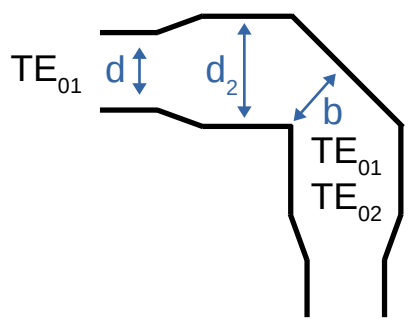

(b)
Fig. 5: Mitre bends structures, (a) pure $\mathrm{TE}_{01}$ mode, and (b) hybrid $\mathrm{TE}_{01}, \mathrm{TE}_{02}$ modes.

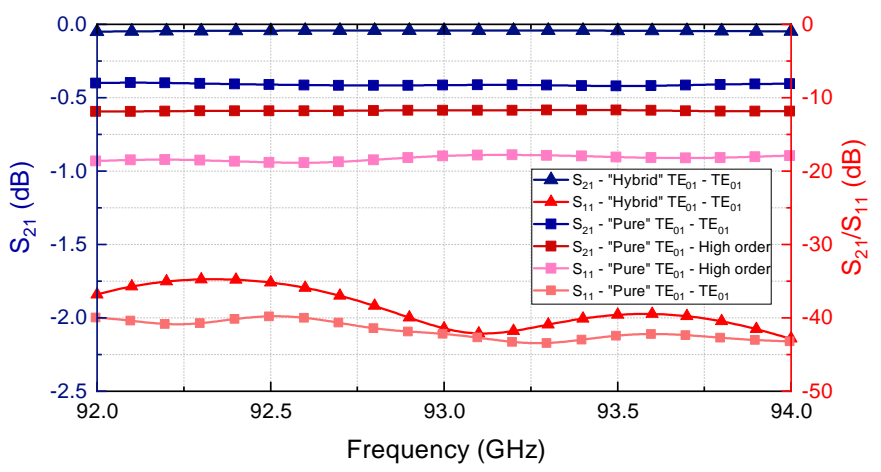

Fig. 6: Transmission parameters of the mitre bend.

From the simulations, a small waveguide diameter shows a large reflection, also the mitre bend will suffer from diffraction losses therefore the diameter of the waveguide needs to be sufficiently large. A further benefit to the enlarged waveguide is in a reduction of the field strength. Typically, a diameter at least a few times, $\sim 5$, the size of the guide wavelength is used. Considering the mitre bend with $d=5 \lambda$ the simulations show a transmission of the $\mathrm{TE}_{01}$ mode around the $-0.4 \mathrm{~dB}$ level and reflection better than $-30 \mathrm{~dB}$ (Fig 6).

For a practical system with multiple bends, of a similar design, which provides the flexibility to transport the radiation over a required distance, the diffraction losses can be significant. Further investigation showed that the diffraction losses can be significantly reduced through introducing a mode conversion before the mitre bend, for example, to convert from

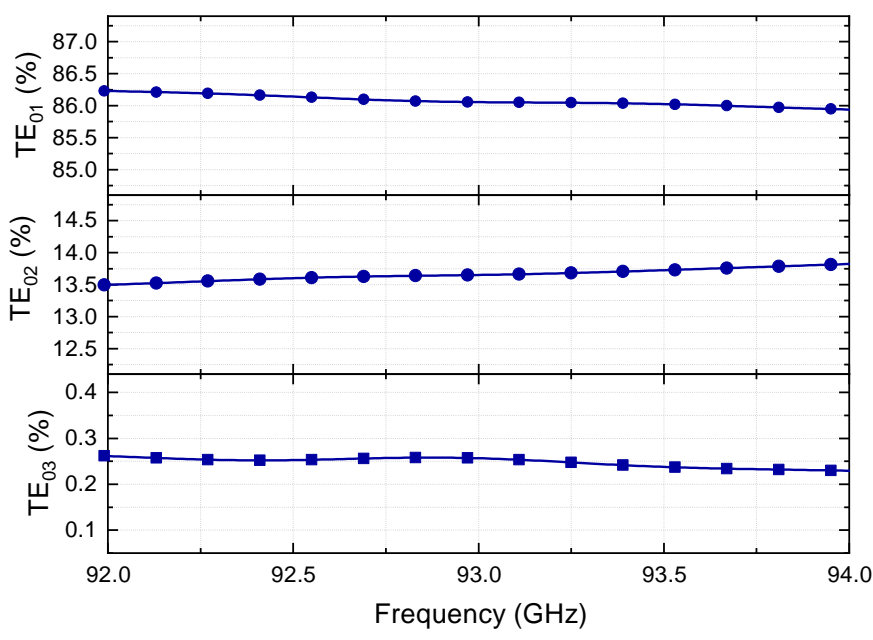

Fig. 7: Simulated mode conversion caused by a linear waveguide taper.

pure $\mathrm{TE}_{01}$ to a mixture of higher order $\mathrm{TE}_{0 n}$ modes, which helps to locate the wave energy more centrally. The conversion can be made through introducing a change of the waveguide diameter. The profile can be determined speedily through optimisation techniques, such as particle swarm optimisation [24], however for simple manufacture a smoothly tapered waveguide transition (Fig. 5(b)), was studied.

The connection between two waveguides with different diameters can be made through a tapered tube. However, it is important to ensure any geometry discontinuities are avoided as these would cause unintended mode conversions. The simplest method is to use the smooth linear taper, the angle of it determines the strength of the conversion to higher order modes $\mathrm{TE}_{0 n}$. Simulations showed that the mode conversion to $\mathrm{TE}_{02}$ is $4 \%, 8 \%, 13.5 \%$, and $20 \%$ when the taper angle was 4, 6, 8 and 10 respectively. An angle of 8 degrees was chosen which resulted in a mode mixture of $86 \% \mathrm{TE}_{01}, 13 \% \mathrm{TE}_{02}$ and $0.2 \% \mathrm{TE}_{03}$ into the mitre bend, as shown in Fig. 7. The initial waveguide diameter had a ratio to wavelength which was set as 5.0 and length of $18.50 \mathrm{~mm}$.

However, the disadvantage for the linear taper is that in the case of a large diameter difference, a long waveguide taper is required. A nonlinear taper, for example with a sinesquare function profile [25], will achieve better performance to maintain the mode content while reducing the taper length by a factor of 2, however it increases the machining complexity. Therefore, the final design of the waveguide taper will be a balance between its length and machining complexity.

The waveguide taper in the mode converter uses the linear taper. It must be at a specific distance $\left(L_{p h}\right)$ from the mitre bend in order to properly phase the modes at the point of the bend. A relatively large taper angle of 8.3 degrees could be used to make a suitable amount of mode conversion to $\mathrm{TE}_{0 n}$ modes. Its accurate length was obtained from a parameter sweep in the numerical simulation. The mode mixture at the central frequency was $86 \% \mathrm{TE}_{01}, 13.5 \% \mathrm{TE}_{02}$ and $0.25 \%$ $\mathrm{TE}_{03}$ (Fig. 7).

In the optimized case, this hybrid mitre bend (Fig. 5 (b)) has an input diameter of $d=5 \lambda_{0}$, output $d_{2}=6.2 \lambda_{0}$ with taper angle 


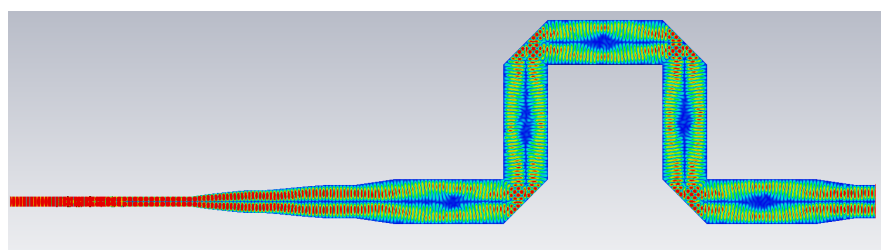

Fig. 8: Simulation model of, and wave propagation through, the W-band transmission line system.

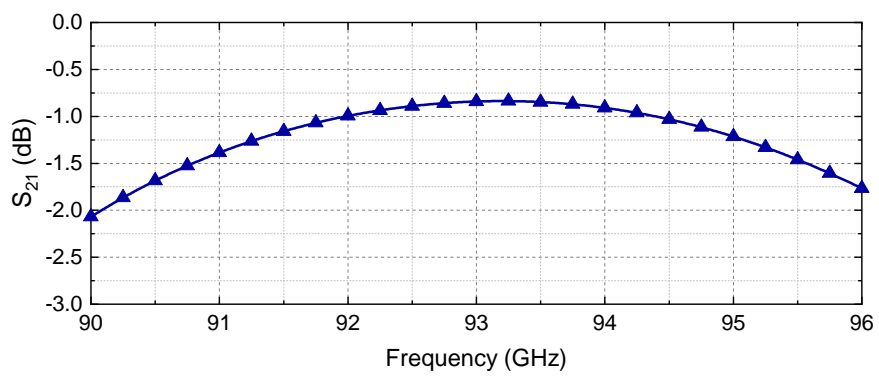

Fig. 9: Simulated W-band transmission line system and inset the simulation model.

of 8.3 degrees which is set away from the mitre bend at $L_{p h}$ $=16 \lambda_{0}$. Therefore, the hybrid mitre bend shows an optimized transmission with an average of $-0.04 \mathrm{~dB}$ transmission over 92-94 GHz.

\section{SIMULATION RESULTS OF W-BAND TRANSMISSION LINE SYSTEM}

A W-band gyro-TWA was developed at the University of Strathclyde. It achieved an output power of $3.4 \mathrm{~kW}$ with the gain of 36-38 dB at a center frequency of $93 \mathrm{GHz}$ [12]. A transmission line system based on the method presented in this paper was designed to transport the millimeter wave radiation about $20 \mathrm{~m}$ away to the application region. A subsection of this system was investigated with an overall length of $0.4 \mathrm{~m}$ with 4 waveguide bends. The optimized mitre bends had an input diameter $d$ of $16.00 \mathrm{~mm}$, phasing diameter $d_{2}$ of $21.40 \mathrm{~mm}$. The taper angle was 8.3 degrees and the phasing length was $18.50 \mathrm{~mm}$. Each of the waveguide bends, when analyzed individually, has a maximum transmission loss of $-0.05 \mathrm{~dB}$ with reflection of under $-30 \mathrm{~dB}$.

The simulated geometry (Fig. 8) comprised of mode converter, taper and four mitre bends separated with smooth guides. To reduce the simulation time, the geometry was simplified; long smooth waveguides were separated into short waveguide sections. The waveguide wall was set with the poor conductivity of $1.0 \times 10^{6} \mathrm{~S} / \mathrm{m}$, the effect is to simulate a waveguide length of $0.4 \mathrm{~m}$ that is the equivalent of $20 \mathrm{~m}$ of oxygen free high conductivity (OFHC) copper. The overall loss was $0.84 \mathrm{~dB}$ at the center frequency (Fig. 9). This result shows that long lengths of this transmission line are viable for use with a $\mathrm{kW}$ level gyro-TWA. It has been shown that conversion of the $\mathrm{TE}_{01}$ to $\mathrm{TE}_{11}$ mode in the overmoded waveguide can be made with high efficiency [26]. This would allow the addition of a $\mathrm{TE}_{11}$ to the $\mathrm{HE}_{11}$ mode converter, such as the corrugated [27] waveguide or a smoothly profiled horn [28].

\section{DISCUSSION AND CONCLUSION}

In many applications there is a requirement to transport the millimeter-wave signal across long distances. In this paper a transmission line system operating with a $\mathrm{TE}_{01}$ mode was studied to meet those needs. The design of the major components, including the mode converter, waveguide taper and mitre bend was presented. The frequency independent design process is applicable to different frequencies for different applications. Each component was analysed as a stand-alone item to achieve an optimal design with consideration of the manufacturing process. The mitre bend geometry was analysed with a pure $\mathrm{TE}_{01}$ input and with additional higher order $\mathrm{TE}_{0 n}$ modes. It was found that the inclusion of those modes resulted in a transmission loss reduction of 10 times when compared to the pure $\mathrm{TE}_{01}$ design. The guides between mitre bends is also very low loss. When considering a conductivity of $5.8 \times 10^{7}$ $\mathrm{S} / \mathrm{m}$ for the metallic waveguide, the Ohmic loss is about 0.006 $\mathrm{dB} / \mathrm{m}$ at a waveguide diameter of $21.40 \mathrm{~mm}$ and frequency of $93 \mathrm{GHz}$. To compare to the corrugated waveguide a $110 \mathrm{GHz}$ transmission line was designed for ITER with a calculated loss of $0.002 \mathrm{~dB} / \mathrm{m}$ at the diameter of $31.75 \mathrm{~mm}$ [6]. The full system, with each component and a series of mitre bends, showed that the system is able to transport the output from a $\mathrm{kW}$ level millimetre wave source over a very long distance.

At the optimal design, the $\mathrm{TE}_{11}$ to $\mathrm{TE}_{01}$ mode converter achieved a central frequency conversion efficiency of $99.1 \%$ while maintaining a better than $-35 \mathrm{~dB}$ return loss. The mitre bend with the higher order mode mixture showed an average of $0.04 \mathrm{~dB}$ transmission loss over 92-94 GHz. As an example, a transmission line system that would be situated at the output of a W-band gyro-TWA was designed. The circular polarized $\mathrm{TE}_{11}$ mode, which is the output mode from the gyro-TWA, was used as the input to the system. With an equivalent overall length of $20 \mathrm{~m}$ and four mitre bends, the transmission loss was about $0.84 \mathrm{~dB}$, at $93 \mathrm{GHz}$, when the waveguide was OFHC copper. An extended bandwidth of $90-96 \mathrm{GHz}$ was studied and the system showed a maximum loss of $-2 \mathrm{~dB}$.

\section{REFERENCES}

[1] G. M. Smith, et al., "Instrumentation for high sensitivity, high power, millimetre wave, electron paramagnetic resonance," IET Colloquium on Millimetre-Wave and Terahertz Engineering \& Technology 2016, London, 2016, pp. 1-5. doi: 10.1049/ic.2016.0017

[2] F. Leuterer, et al., "Experience with the ECRH system of ASDEXupgrade," Fusion Eng. Des., vol. 53, no. 1, pp. 485-489, 2001. doi: 10.1016/S0920-3796(00)00524-X

[3] P. J. B. Clarricoats and A. D. Olver, Corrugated horns for microwave London, U.K.: Peregrinus, 1984. doi: 10.1049/PBEW018E

[4] E. J. Kowalski, D. S. Tax, M. A. Shapiro, J. R. Sirigiri, and R. J. Temkin, "Linearly polarized modes of a corrugated metallic waveguide," IEEE Trans. Microw. Theory Techn., vol. 58, no. 11, pp. 2772-2780, Nov. 2010. doi: 10.1109/TMTT.2010.2078972

[5] L. Zhang, W. He, J. Clarke, K. Ronald, A. D. R. Phelps and A. W. Cross, "Systematic study of the corrugated waveguide as a microwave undulator," J. Synchrotron Rad., vol. 26, no. 1, pp. 11-17, 2019. doi: $10.1107 /$ S 1600577518014297

[6] J. L. Doane, "Design of circular corrugated waveguides to transmit millimeter waves at ITER," Fusion Sci. Tech., vol. 52, no. 1, pp. 159173, 2008. doi: 10.13182/fst08-a1662

[7] P. P. Woskov, V. S. Bajaj, M. K. Hornstein, R. J. Temkin and R. G. Griffin, "Corrugated waveguide and directional coupler for CW 250-GHz gyrotron DNP experiments," IEEE Trans. Microwave Theory Techn., vol. 53, no. 6, pp. 1863-1869, June 2005. doi: 10.1109/TMTT.2005.848097 
[8] A. A. Bogdashov, and G. G. Denisov, "Asymptotic theory of highefficiency converters of higher-order waveguide modes into eigenwaves of open mirror lines," Radiophys. Quantum Electron., vol. 47, no. 4, pp. 283-296, Apr. 2004. doi: 10.1023/B:RAQE.0000047649.17664.6e

[9] V. I. Belousov, A. A. Bogdashov, A. V. Chirkov, G. G. Denisov, and S. V. Kuzikov, "New components for $\mathrm{TE}_{01}$ transmission lines," Strong Microwaves in Plasmas, vol. 2, pp.948-954, 1999. ISBN: 5804800086, 9785804800087

[10] A. A. Bogdashov, et al., "High-power Ka-band transmission line with a frequency bandwidth of $1 \mathrm{GHz}$, , Radiophys. Quantum Electron., vol. 58, no. 10, pp. 777-788, March, 2016. doi: 10.1007/s11141-016-9650-5

[11] A. A. Bogdashov, G. G. Denisov, D. Lukovnikov, Yu. Rodin, and J. Hirshfield, "Ka-band resonant ring for testing components for a highgradient linear accelerator," IEEE Trans. Micro. Theory Techn., vol. 53, no. 10 , pp. 3152-3155, Oct. 2005. doi: 10.1109/TMTT.2005.855357

[12] W. He, C. R. Donaldson, L. Zhang, K. Ronald, A. D. R. Phelps, and A. W. Cross, "Broadband amplification of low-terahertz signals using axis-encircling electrons in a helically corrugated interaction region," Phys. Rev. Lett., vol. 119, 184801, 2017. doi: 10.1103/PhysRevLett.119.184801.

[13] L. Zhang, C. R. Donaldson, P. Cain, A. W. Cross, and W. He, "Amplification of frequency-swept signals in a W-band gyrotron travelling wave amplifier," IEEE Electron Device Lett., vol. 39, no. 7, pp. 1077-1080, May 2018. doi: 10.1109/LED.2018.2836868

[14] G. Liu, et al., "A millimetre wave high-order $\mathrm{TE}_{13}$ mode converter," IEEE Trans. Electron Dev., vol. 63, no. 7, pp. 2907-2911, May 2016. doi: 10.1109/TED.2016.2562025.

[15] D. A. Constable, X. S. Frampris, K. Ronald, W. He, C. G. Whyte, and C. W. Robertson, "A novel cylindrical TE 21 mode converter," Rev. Sci. Instru., vol. 81, no. 9, 094702, 2010. doi: 10.1063/1.3480994

[16] Y. Wang, et al., "Wideband circular $\mathrm{TE}_{21}$ and $\mathrm{TE}_{01}$ mode converters with same exciting topologies," IEEE Trans. Electron Dev., vol. 23, no. 10, pp. 4088-4095, Aug. 2016. doi: 10.1109/TED.2016.2596785.

[17] C. Schulz, C. Baer, T. Musch, I. Rolfes, and B. Will, "Investigation of a circular $\mathrm{TE}_{11}-\mathrm{TE}_{01}$-mode converter in stepped waveguide technique," Int. J. Microw. Wireless Technol., vol. 7, no. 3-4, pp. 229-237, June 2015. doi: 10.1017/S1759078715000203.

[18] G. G. Denisov, G. I. Kalynova, and D. I. Sobolev, "Method for synthesis of waveguide mode converters," Radiophys. Quantum Electron., vol. 47, no. 8, pp. 615-620, Aug. 2004. doi: 10.1023/B:RAQE.0000049559.74097.86

[19] M. Thumm, "High-power millimeter-wave mode converters in overmoded circular waveguides using periodic wall perturbations," Int. J. Electron., vol. 57, no. 6, pp. 1225-1246, 1984. doi: $10.1080 / 00207218408938998$

[20] L. Zhang, W. He, J. Clarke, K. Ronald, A. D. R. Phelps, and A. W. Cross, "Microwave undulator using a helically corrugated waveguide," IEEE Trans. Electron Devices, vol. 65, no. 12, pp. 5499-5504, Oct. 2018. doi: 10.1109/TED.2018.2873726

[21] L. Zhang, et al., "Multi-mode coupling wave theory for helically corrugated waveguide," IEEE Trans. Microw. Theory Techn., vol. 60, no. 1, pp. 1-7, Nov. 2012. doi: 10.1109/TMTT.2011.2170848.

[22] G. G. Denisov, V. L. Bratman, A. D. R. Phelps, and S. V. Samsonov, "Gyro-TWT with a helical operating waveguide: New possibilities to enhance efficiency and frequency bandwidth," IEEE Trans. Plasma Sci., vol. 26, no. 3, pp. 508-518, Jun. 1998. doi: 10.1109/27.700785.

[23] D. B. McDermott, J. Pretterebner, C. K. Chong, C. F. Kinney, M. M. Razeghi, N. C. Luhmann, "Broadband linearly polarized beat-wave $\mathrm{TE}_{m 1} / \mathrm{TE}_{11}$ mode converters," IEEE Trans. Micro. Theory Techn., vol. 44, no. 2, pp. 311-317, Feb. 1996, doi: 10.1109/22.481581

[24] A. A. Bogdashov, and Rodin Yu., "Mode converter synthesis by Particle Swarm Optimization," Int. J. Infrared Milli. Waves, vol. 28, pp. 627-638, 2007. doi: 10.1007/s10762-007-9248-2

[25] L. Zhang, et al., "Optimization and measurement of a smoothly profiled horn for a W-Band gyro-TWA," IEEE Trans. Electron Devices, vol. 64, no. 6, pp. 2665-2669, April 2017. doi: 10.1109/TED.2017.2687949

[26] A. Patel, et al., "Millimeter-wave $\mathrm{TE}_{01}-\mathrm{TE}_{11}-\mathrm{HE}_{11}$ mode converter using overmoded circular waveguide," J. Electro. Waves Appl., vol. 32, pp. 1768-1778, 2018. doi: 10.1080/09205071.2018.1468286

[27] P. McElhinney, et al., "An output coupler for a W-Band high power wideband gyroamplifier," IEEE Trans. Electron Dev., vol. 64, no. 4, pp. 1763-1766, June 2017. doi: 10.1109/TED.2017.2660304

[28] L. Zhang, et al., "Optimization and measurement of a smoothly profiled horn for a W-Band gyro-TWA," IEEE Trans. Electron Dev., vol. 64, no. 6, pp. 2665-2669, June 2017. doi: 10.1109/TED.2017.2687949 